Riihimaa, J. J. (1964a), High resolution spectral observations of Jupiter's decametric radio emission, Nature 202, 476-477.

Riihimaa, J. J. (1964b), Observations of the fine structure of Jupiter's decametric radio emission, Ann. Acad. Sci. Fennicae, Ser. A VI 156.

Rishbeth, H. (1959), The ionosphere of Jupiter, Australian J. Phys. 12, 466-468.

Scarf, F. L., W. Bernstein, and R. W. Fredricks (1965), Electron acceleration and plasma instabilities in the transition region, J. Geophys. Res. 70, No. 1, 9-20.

Shain, C. A. (1956), 18.3 Mc/s radiation from Jupiter, Australian J. Phys. 9, 61-73.

Sherrill, W. M. (1965), Polarization of Jovian emission at decametric wavelengths, Nature 205, 270-271.

Sherrill, W. M., and M. P. Castles (1963), Survey of the polarization of Jovian radiation at decametric wavelengths, Astrophys. J. 138, $587-598$.

Slee, O. B., and C. S. Higgins (1964), Long baseline interferometry of Jovian decametric radio bursts, Nature 197, 781-782.

Warwick, J. W. (1961), Theory of Jupiter's decametric radio emissions, Ann. N. Y. Acad. Sci. 95, 39.

Warwick, J. W. (1963a), Dynamic spectra of Jupiter's decametric emission, 1961, Astrophys. J. 137, 41-60.

Warwick, J. W. (1963b), Repeatibility of Jupiter's decametric radio emissions, Science 140, 814-816.

Zelezniakov, V. V. (1960), The stability of a magnetoactive perturbations, Radiophysica 3, 57 .

\section{Additional Related Reference}

Roberts, J. A. (1963), Radio emission from the planets, Planet. Space Sci. 1 1, 221-259.
Discussion Following Ellis's Paper

J. W. Warwick: Have you a deductive theory of Jupiter's magnetosphere?

Answer: Yes, the scale height is the sensitive parameter of the theory, and for Jupiter must correspond to $5000{ }^{\circ} \mathrm{K}$ instead of the $1000{ }^{\circ} \mathrm{K}$ of the Earth's magnetosphere.

S. Silver: Is there reason to suppose that there is a magnetosphere structure around Jupiter?

Answer: Yes. It depends on the temperature.

C. Sagan: What about Jupiter V, the innermost satellite?

Answer: There may be possible effects, depending on the electron density profile in the magnetosphere.

$J$. A. Roberts: The field lines intersecting Jupiter V may be too low in latitude to correspond to observable effects.

C. Sagan: Does the existence of a satellite atmosphere fit your theory?

Answer: Yes.

I. Shapiro: Does Io rotate fast enough to have its own field?

Answer (by C. Sagan): Maps of the Galilean satellites exist and show that they all rotate synchronously.

\title{
Results of Recent Investigations of Jupiter's Decametric Radiation
}

\author{
T. D. Carr, S. Gulkis, A. G. Smith, J. May, G. R. Lebo, D. J. Kennedy, and H. Bollhagen \\ Department of Physics and Astronomy, University of Florida, Gainesville, Fla.
}

\begin{abstract}
The activity of Jupiter's decametric radiation appears to be greatest between 5 and $10 \mathrm{Mc} / \mathrm{s}$, but measurements made below $10 \mathrm{Mc} / \mathrm{s}$ are subject to large ionospheric errors. No significant change in rotation period has appeared since 1960. The effect of the satellite Io as reported by Bigg has been corroborated. Marked variations in axial ratio with System III longitude were observed, from which estimates were made of the meridians of the poles. A ray-tracing study was made of the focusing of radiation escaping from possible Jovian field-alined ducts. The effect of asymmetrical stop zones is discussed. A possible explanation of the influence of Io is offered.
\end{abstract}

\section{Introduction}

Systematic observations of the decametric radiation from Jupiter have been conducted by the University of Florida group at Gainesville since 1956, and at the Maipu Radioastronomical Observatory in Chile since 1959. Monitored recordings are regularly made with simple calibrated radiometers at several fixed frequencies between 5 and $52 \mathrm{Mc} / \mathrm{s}$. Intensities of the circular polarization components are measured at three of the frequencies. Recordings of the dynamic spectra and detailed structure of bursts are made during the more intense noise storms. The purpose of this paper is to present some of the more recent results of the observational program, and of related theoretical investigations.

\section{Average Spectra}

One of the objectives of the program has been the determination of the average spectral distribution of the decametric radiation occurring during an entire apparition. Such a spectrum is shown in figure 1 . 




FigURE 1. Spectral distribution of Jupiter's radio emission. The decameter-wavelength curves are for 1961.

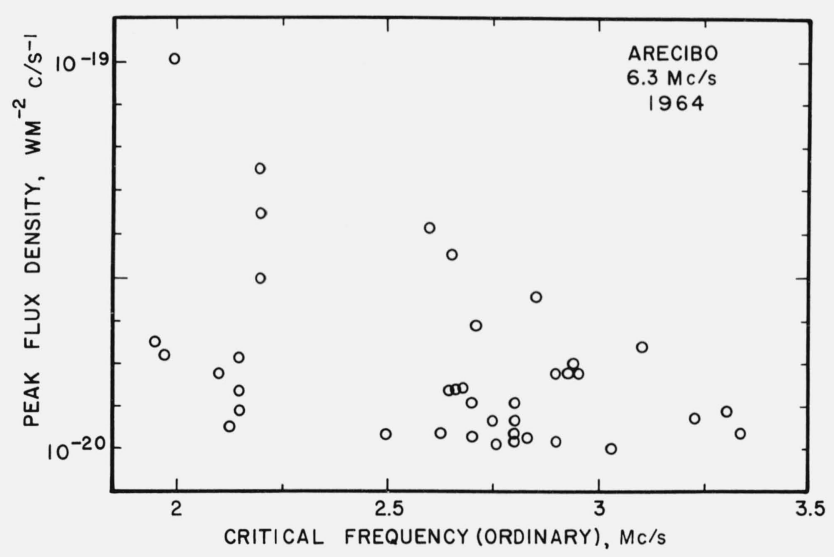

FIGURE 2. Effect of critical frequency upon flux density of noise bursts.

In order to illustrate the relationship between the decameter-wavelength region and the other parts of Jupiter's radio spectrum, the decimeter-wavelength and thermal regions have also been included. Since the decameter radiation is highly sporadic, the curve for the peak flux densities lies considerably above that for the average.

There is considerable uncertainty regarding the spectra below $10 \mathrm{Mc} / \mathrm{s}$, due to the proximity of the ionospheric critical frequency. The smooth curves for the decameter region were obtained in 1961 [Carr et al., 1964]; points obtained more recently are also shown. The maximum activity, as observed at ground level between midnight and dawn during the years of minimum sunspot number, apparently occurs between 5 and $10 \mathrm{Mc} / \mathrm{s}$. Whether or not future observations to be made from above the ionosphere will reveal that the true maximum lies at a significantly lower frequency than this remains to be seen.
Figure 2 suggests that the true maximum could indeed occur at a lower frequency. This figure is a scatter diagram of the peak flux densities of a series of Jupiter noise bursts at $6.3 \mathrm{Mc} / \mathrm{s}$ as a function of the $F$ region critical frequency for the ordinary mode in the terrestrial ionosphere. Jupiter was near the zenith at the times of observation, and the sunspot number was close to its minimum. The figure suggests that ionospheric attenuation of the $6.3-\mathrm{Mc} / \mathrm{s}$ signals was quite severe whenever the critical frequency exceeded the relatively low value of $2.5 \mathrm{Mc} / \mathrm{s}$. It would therefore seem that flux density measurements from beneath the ionosphere at frequencies less than $10 \mathrm{Mc} / \mathrm{s}$ can serve only as lower limits, unless adequate correction can be made for attenuation.

\section{Periodicities}

Although the occurrence of the Jovian decameter radiation appears superficially to be random, statistical analyses have revealed three pronounced periodicities. These are (a) the System III rotation period, (b) the period of the satellite Io in its orbital motion about Jupiter, and (c) the 11-year period of the sunspot cycle.

Plots of the probability of occurrence of the radiation as a function of the System III longitude of the central meridian usually indicate three major source zones, as shown in figure 8 . The sources are easily recognizable at the higher frequencies. They grow wider as the frequency is reduced, and below about $15 \mathrm{Mc} / \mathrm{s}$ become poorly defined. Their positions appeared to remain fixed in terms of System III longitude until 1960. Since 1960, however, they have drifted toward higher longitudes at the rate of about $11^{\circ}$ per year. The implication is that the rotation period of the radio sources increased rather abruptly by about 1 sec in 1960, and has remained essentially constant since that time. These results have been presented in detail by Smith et al., [1965]. More recent results, through the 1964 apparition, indicate no further change in the rotation period.

A striking correlation of the Jovian decametric activity with the position of the satellite Io was discovered by Bigg [1964]. The recent reexamination of all the Florida and Chile data has resulted in the complete corroboration of Bigg's findings. These results, together with evidence for a similar but less pronounced influence by the satellites Gannymede and Europa, have been presented by Lebo et al., [1965].

It has long been known that an inverse correlation exists between the yearly averages of Jupiter activity and sunspot number. Recently obtained results from the 1964 apparition indicate a continued rise in Jupiter activity. The minimum in Jupiter activity lagged the sunspot maximum by about a year. It will be of interest to determine whether a similar lag exists between the Jupiter activity maximum and the sunspot minimum. 


\section{Polarization}

Extensive measurements of the intensities of the right and left circular components of the Jupiter radiation have been made at the University of Florida and in Chile. Typical noise bursts are a second or so in duration. The apparent axial ratio, $\alpha$, which is measured for each burst, is given by

$$
\alpha=\frac{S_{L}^{1 / 2}-S_{R}^{1 / 2}}{S_{L}^{1 / 2}+S_{R}^{1 / 2}}
$$

In this expression $S_{L}$ and $S_{R}$ are the flux densities of the left and right circular components of the burst peak, not including the contribution from the galactic background. The magnitude of the apparent axial ratio is less than or equal to that of the true axial ratio, being less if an unpolarized component is present. There is usually a fairly large random variation in the values of $\alpha$ for successive bursts; however, it has been found that smoothing can reveal significant variations.

Carr et al., [1961] demonstrated that at $22.2 \mathrm{Mc} / \mathrm{s}$ the algebraic averages of $\alpha$ for the three major source zones varied slightly but significantly. The right circular component was predominant for all three, but source C displayed the greatest tendency toward lefthandedness. Dowden [1963] showed that there was a pronounced variation in the smoothed values of $\alpha$ with respect to longitude at $10 \mathrm{Mc} / \mathrm{s}$, the polarization sense actually changing with longitude.

Figure 3 shows some results of observations at 22.2 $\mathrm{Mc} / \mathrm{s}$ in Florida and in Chile during 1962 and 1963. The algebraic averages of $\alpha$ over $20^{\circ}$ longitude intervals are plotted with respect to System III longitude.


FIGURE 3. Variation of polarization with longitude at $22.2 \mathrm{Mc} / \mathrm{s}$. Bar heights indicate standard deviation for a single measurement.
Although $\alpha$ definitely became positive in a region of low activity near $360^{\circ}$, this portion of each curve is rather poorlv defined.

Figure 4 illustrates the same type of results for 15.8-Mc/s observations made in Chile during 1962 and 1963. It should be noted that these two curves are remarkably similar, except that the one for 1963 is displaced $10^{\circ}$ to $20^{\circ}$ toward higher longitudes relative to that for 1962. This displacement is presumably due to the slight error in the assumed rotation period.

Preliminary results at $10 \mathrm{Mc} / \mathrm{s}$ are presented in figure 5 . The curve in this case was obtained from the observations of only 1 month; it is nevertheless surprisingly regular.

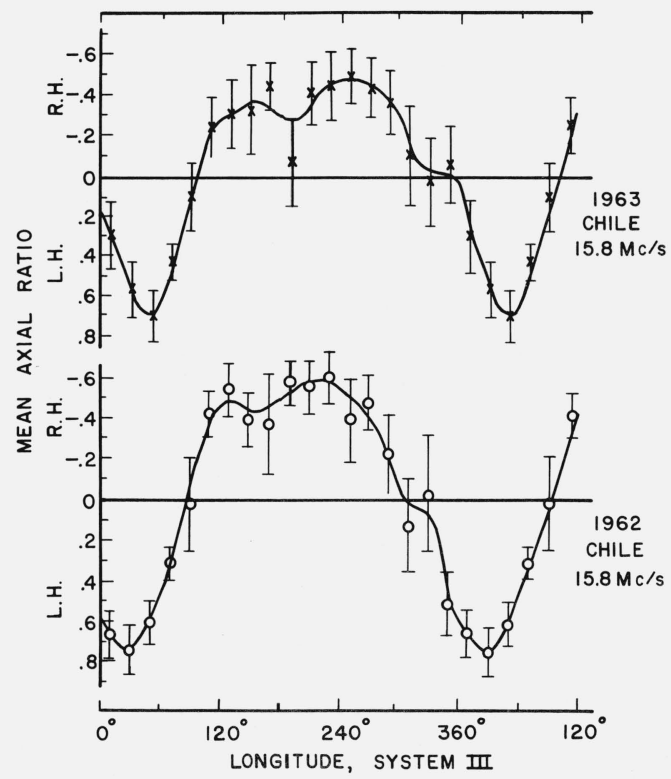

FIGURE 4. Variation of polarization with longitude at $15.8 \mathrm{Mc} / \mathrm{s}$. Bar heights indicate standard deviation for a single measurement.



FigURE 5. Variation of polarization with longitude at $10 \mathrm{Mc} / \mathrm{s}$. Bar heights indicate standard deviation for a single measurement. 
An averaged curve for each of the three frequencies is shown in figure 6 , correction having been made for the yearly longitude drift. It is seen that the longitude zone in which the polarization sense is left hand widens as the frequency is reduced, until at $10 \mathrm{Mc} / \mathrm{s}$ it is almost $180^{\circ}$ wide.

Figure 7 shows the distribution of apparent axial ratio values of individual bursts at $10,15.8$, and 22.2 $\mathrm{Mc} / \mathrm{s}$. Although the most probable axial ratio values were about \pm 0.55 for $10 \mathrm{Mc} / \mathrm{s}$ and about -0.45 for the higher frequencies, the values \pm 1.0 occurred relatively often. This figure clearly shows the increase in the relative number of left-hand bursts which occurs when the frequency is decreased.

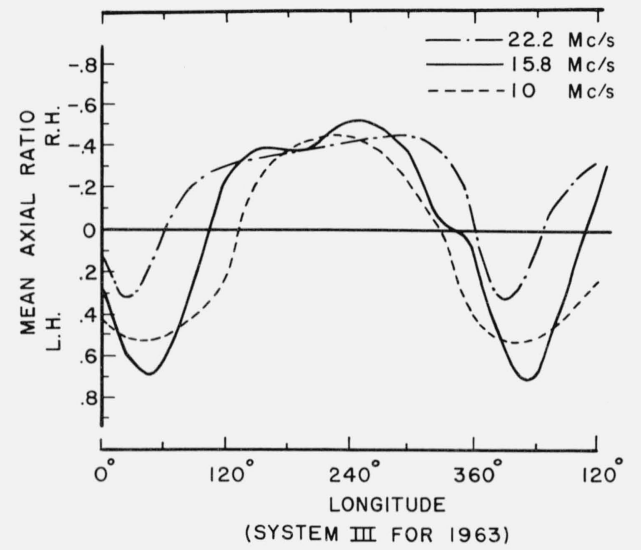

FIGURE 6. Smoothed curves showing longitude variation of polarization at three frequencies.

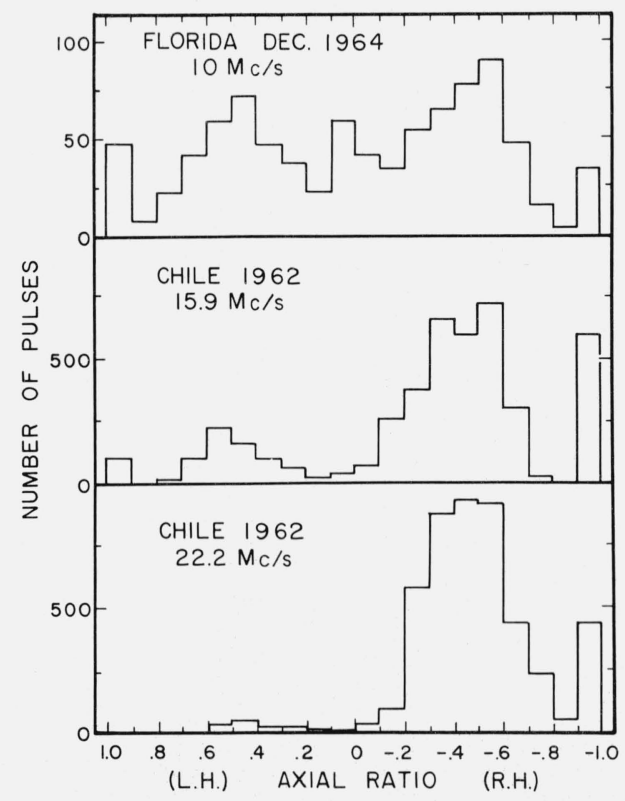

FIGURE 7. Distributions of axial ratio values.
In several of the theories which have been suggested to account for the radiation, emission occurs in the extraordinary mode, at or near the local electron gyrofrequency. The magnetic field is assumed to be that of a dipole which is inclined about $10^{\circ}$ with respect to the rotational axis and is offset from the center of the planet. Assuming that no reflections occur, that the extraordinary mode remains predominant, and that the early propagation is more or less along the field lines, certain generalizations can be made regarding the magnetic poles. One can conclude that the fieldsource and field-sink poles must lie near the longitudes at the centers of the regions of right-hand and left-hand polarization, respectively, in figure 6 . The System III longitude of the field-source pole in 1963 would thus have been about $215^{\circ}$, and that of the fieldsink pole about $35^{\circ}$. Observations of the synchrotron radiation at decimeter wavelengths have indicated that the geographic north and south poles are at System III longitudes of about $200^{\circ}$ and $20^{\circ}$, respectively. Thus the magnetic poles on Jupiter appear to be oriented oppositely, relative to the geographic poles, from the way they are on earth. The highest frequency at which the decametric radiation has been obtained is about $40 \mathrm{Mc} / \mathrm{s}$. It is clear from figure 6 that any radiation occurring at $40 \mathrm{Mc} / \mathrm{s}$ must have been polarized in the right-hand sense. We can thus conclude that the surface value of the magnetic field intensity at the field-source pole is at least $14 \mathrm{G}$. On the other hand, figure 6 suggests that the cutoff frequency for the left circular component is not far from $28 \mathrm{Mc} / \mathrm{s}$, indicating that the field at the sink pole is at least $10 \mathrm{G}$. The magnetic dipole must therefore be displaced from the center of the planet toward the field-source pole, as was also concluded by Dowden [1963].

Figure 8 shows the relationship between the longitude distributions of occurrence probability and apparent axial ratio. The assumed longitudes of the source and sink poles are indicated. The meridian of the field-source pole passes between radiation sources A and B. Source C seems to lie largely in the opposite hemisphere, but its center does not occur very close to the meridian of the field-sink pole.

Polarization measurements of the radiation from Jupiter at $6.3 \mathrm{Mc} / \mathrm{s}$ were made in 1964 from a deep valley near the Arecibo Ionospheric Observatory in Puerto Rico. Despite the shielding afforded by the valley walls, and the prevailing near-optimum conditions for propagation through the ionosphere, most of the recordings were unusable because of excessive atmospheric noise from local thunderstorms. Nevertheless, some interesting effects were observed. On one occasion, an unusually long Jovian noise storm persisted from before dawn until after sunrise. At the start, the right circular component was stronger than the left. However, as the critical frequency increased, the right circular component grew progressively weaker relative to the left circular component, as shown in figure 9. The effect was presumably caused by the selective absorption of the right circular (extraordinary) component as the ionization increased. 


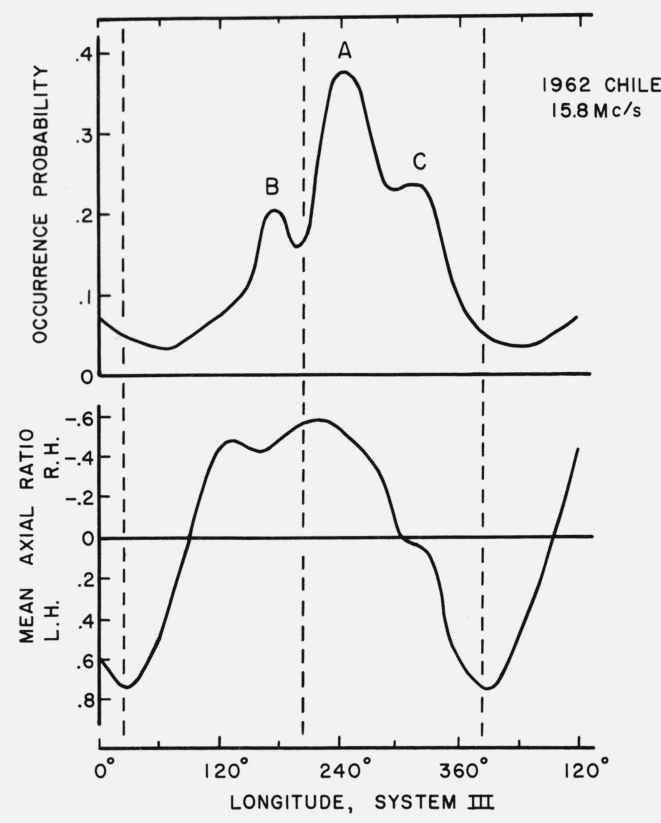

FIGURE 8. Smoothed curves showing variation of axial ratio and occurrence probability with longitude. Vertical dashed lines indicate assumed longitudes of poles.

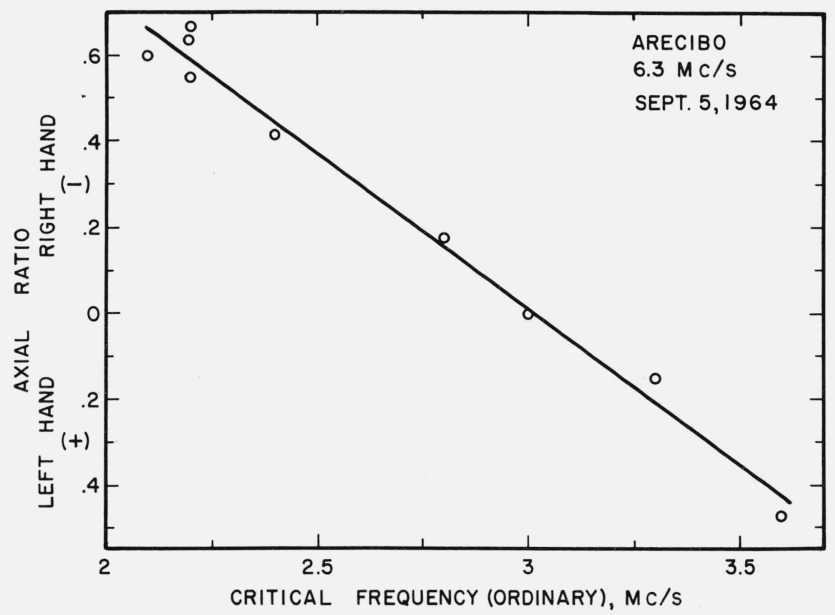

FigURE 9. Effect of critical frequency upon axial ratio.

This bears out the earlier conclusion that results of observations at the lower frequencies are likely to be misleading if corrections for ionospheric effects are not made.

Another interesting observation made at Arecibo was that the noise bursts apparently lengthen when the frequency is lowered sufficiently. The durations of most of the bursts at $6.3 \mathrm{Mc} / \mathrm{s}$ ranged from about 10 to $100 \mathrm{sec}$, while at $10 \mathrm{Mc} / \mathrm{s}$ or higher, the majority were between 1 and 10 sec in length.

\section{Theoretical Studies of Propagation in the Jovian Magnetosphere}

Carr [1962] has suggested that field-alined layers of slightly enhanced ionization in Jupiter's magnetosphere may strongly influence the propagation of the radiation produced at high latitudes. Rays which were initially trapped beneath a layer would escape from the duct when the transverse electron density gradient became insufficient to bend the ray along the field line. A partial focusing would occur upon escape, and rays of different frequencies would escape in slightly different directions. This effect, combined with the rotation of the planet and assumed departures of the field configuration from that of a symmetrically oriented dipole, could account qualitatively for several of the observed phenomena.

Gulkis [1965] recently carried out a quantitative investigation of the focusing of the radiation escaping from a field-alined duct. He used a ray-tracing procedure patterned after that employed by Yabroff [1961] for whistler studies. A dipole field with its axis tipped $10^{\circ}$, and a magnetosphere containing a duct having a Gaussian profile were assumed. Ray tracings were made for each of many combinations of frequency, initial wave normal direction, ambient electron density distribution, magnetic field intensity, and the strength, thickness, and location of the duct. Duct enhancement factors as low as 20 or 30 percent were found to be effective. Focusing occurred for certain seemingly plausible combinations of the adjustable parameters. A change in frequency of more than 1 or $2 \mathrm{Mc} / \mathrm{s}$ was necessary in order to change the direction of the focused rays appreciably; it appears significant that this is the approximate bandwidth of the observed noise bursts. Calculations indicated that the presence of the duct increased the intensity of the radiation in the direction of focusing by about $12 \mathrm{~dB}$ above what it would have been without the duct.

As expected, it was found that as either of the poles approached the central meridian, the frequency of the radiation focused toward the earth drifted to higher values. After the pole crossed the central meridian the direction of frequency drift reversed. The calculated rate of frequency drift was of the same order of magnitude as the observed values. Since only the extraordinary mode was assumed to be present initially, waves of opposite senses of elliptical polarization would originate from opposite sides of the magnetic equator. It was concluded that the field-alined duct model could readily be adjusted to predict several of the observed phenomena on a semiquantitative basis.

Radiation generated in a planetary magnetosphere in the extraordinary mode at or below the local electron gyrofrequency normally cannot escape. It is confronted by a stop zone, i.e., a region in which the refractive index is imaginary [Booker, 1962]. For propagation along a field line, the inner stop-zone boundary occurs where the gyrofrequency equals the wave frequency. The thickness of the stop zone depends upon both the magnetic field and the electron 
density; the thickness would decrease if the electron density were decreased or if the magnetic field were increased. The Doppler-shifted gyroradiation in the Ellis-McCulloch [1963] theory can escape provided the shift is to a high enough frequency. In such a case, the region in which the radiation is emitted lies outside the outer stop-zone boundary.

In two of the other emission models which have been proposed, the radiation has been assumed to result from the Cerenkov process and from amplified whistlers, respectively. The stop zone would prevent escape in either case, according to the usual magnetoionic theory. However, Piddington [1960] refers to possible ways in which radiation emitted in the extraordinary mode might penetrate the stop zone in the case of solar radiation. In these processes, the extraordinary waves are assumed to create ordinary waves at the stop zone, and the latter readily escape. If this is indeed taking place in Jupiter's magnetosphere, then our earlier conclusions regarding the types of poles at the two specified longitudes are incorrect. Thus the pole at $215^{\circ}$ longitude would be the field-sink rather than the field-source pole.

Gulkis [1965] has pointed out that for all three models, i.e., Doppler-shifted cyclotron emission, Cerenkov emission, and escaped whistlers, a decrease in the stop-zone thickness would increase the probability of emission. An asymmetrical field could thus give rise to an asymmetrical distribution of the radiation with respect to longitude. This effect might account in part for the longitude dependence of the observed activity.

The remarkable influence of the satellite Io in triggering the radiation from Jupiter may well provide the key leading to the solution of the mystery of the origin of the decameter radiation. In one of the two configurations of maximum emission probability, source B is on the central meridian and the orbital position angle of Io, relative to superior geocentric conjunction, is about $90^{\circ}$ [Bigg, 1964; Lebo et al., 1965]. In the other favorable configuration, source $A$ is on the central meridian and the position angle of Io is about $240^{\circ}$. Io is thus close to the position of maximum elongation in both cases. It is conceivable, although not very likely, that the phenomenon results from magnetospheric tides raised by Io. One would expect two diametrically opposite tidal crests. As each of the troughs between the crests crossed the central meridian, the accompanying decrease in the thickness of the stop zone might allow radiation to escape toward the earth.

We suggest another explanation which is perhaps more plausible. Io travels among the charged particles trapped in Jupiter's Van Allen Belts. We assume that it has acquired an ionosphere of its own, particularly on its windward side. This ionosphere must be highly diamagnetic. The field lines in Jupiter's rotating magnetosphere would this spread apart as they slip past Io, converging to their original positions on the other side. Trapped particles following the bulging field lines would be kept well outside Io's ionosphere, and would not collide with it. How- ever, perturbation of the field near Io might somehow precipitate trapped electrons into Jupiter's ionosphere. One such possibility is that the changing field would accelerate certain electrons and would decelerate others; the lowered mirroring altitude of those accelerated could result in their precipitation.

There might thus be a continual dumping of particles which had been trapped along those field lines passing closest to Io. As Warwick has suggested [1963], the dumping of previously trapped particles into Jupiter's ionosphere might result in the emission of Cerenkov radiation. In Warwick's original model, Cerenkov radiation from dumped electrons is reflected from the top of the ionosphere back toward the earth. However, we will assume that the radiation merely grazes the top of the ionosphere. The various rays from a point source in the region of dumping are refracted to a greater or lesser extent away from the planet, leaving a zone of shadow beneath a sharply defined ray envelope. This bounding surface approaches a wideangle cone at some distance from the planet. The angular width of the cone is only slightly less than $180^{\circ}$. The rays closest to it are essentially parallel. The layer of parallel rays sweeps past the earth when Io is near either of the two positions of maximum elongation, resulting in the observed increases in activity. The observed radiation would come from regions near Jupiter's limb rather than from the socalled "sources" on the central meridian.

It is not clear why such radiation should not be observed every time Io is near maximum elongation. The explanation is very likely closely related to the geometrical effects of dipole tilt, dipole eccentricity, and distortion of the field from that of a true dipole. It seems significant in this connection that for both of the configurations resulting in maximum emission, the pole near $200^{\circ}$ longitude lies between the meridian of Io and the central meridian. Such mirror-image symmetry between the two configurations suggests a simple geometrical explanation based on a tilted dipole. However, it would undoubtedly be complicated by other factors such as stop-zone asymmetries and propagation anisotropy.

The authors express their gratitude to Jorge Levy of the Maipu Radioastronomical Observatory, and to graduate students and staff at the University of Florida Radio Observatory for assistance in equipment construction, observations, and data reduction. Appreciation is also expressed to Claudio Anguita, Director of the National Astronomical Observatory, University of Chile, and to W. E. Gordon, Director of the Arecibo Ionospheric Observatory, for providing research facilities, and to R. J. Armstrong of the University of the West Indies for supplying ionospheric data. The financial support of the National Science Foundation, the U.S. Army Research Office (Durham), the Office of Naval Research, and the National Aeronautics and Space Administration is gratefully acknowledged. 


\section{References}

Bigg, E. K. (1964), Influence of the satellite Io on Jupiter's decametric emission, Nature 203, 1008-1010.

Booker, H. G. (1962), Guidance of radio and hydromagnetic waves in the magnetosphere, J. Geophys. Res. 67, 4135-4162.

Carr, T. D., A. G. Smith, H. Bollhagen, N. F. Six, and N. E. Chatterton (1961), Recent decameter-wavelength observations of Jupiter, Saturn, and Venus, Astrophys. J. 134, 105-125.

Carr, T. D. (1962), The possible role of field-alined ducts in the escape of decameter radiation from Jupiter, presented at NASA Jupiter conference, New York.

Carr, T. D., G. W. Brown, A. G. Smith, C. S. Higgins, H. Bollhagen, J. May, and J. Levy (1964), Spectral distribution of the decameter radiation from Jupiter in 1961, Astrophys. J. 140, 778-795.

Dowden, R. L. (1963), Polarization measurements of Jupiter radio bursts at $10.1 \mathrm{Mc} / \mathrm{s}$, Australian J. Phys. 16, 398 .

Ellis, G. R. A., and P. M. McCulloch (1963), The decametric radio emissions of Jupiter, Australian J. Phys. 16, 380-397.

Gulkis, S. (1965), A theoretical model for the emission of the decametric radiation from Jupiter, Ph.D. dissertation, University of Florida.

Lebo, G. R., A. G. Smith, and T. D. Carr (1965), Jupiter's decametric emission correlated with the longitudes of the first three Galilean satellites, Science 148, 1724-1725.
Piddington, J. H. (1960), Radio Astronomy (Harper and Brothers, New York).

Smith, A. G., G. R. Lebo, N. F. Six, T. D. Carr, H. Bollhagen, J. May, and J. Levy (1965), Decameter-wavelength observations of Jupiter: the apparitions of 1961 and 1962, Astrophys. J. 141, 457-477.

Warwick, J. W. (1963), Dynamic spectra of Jupiter's decametric emission, 1961, Astrophys. J. 137, 41-60.

Yabroff, I. (1961), Computation of whistler ray paths, J. Res. NBS 65D (Radio Prop.), 485-505.

\section{Discussion Following Carr et al.'s Paper}

J. A. Roberts: Are the other satellites over the same part of Jupiter as Io when the low-frequency emission is stimulated?

Answer: Probably not.

\title{
Results From CSIRO, Sydney, Australia
}

\author{
O. B. Slee and C. S. Higgins \\ Radiophysics Laboratory, Commonwealth Scientific and Industrial Research Organization, \\ Sydney, Australia
}

The CSIRO data at $19.7 \mathrm{Mc} / \mathrm{s}$ confirm in general terms the influence of Io reported by Bigg [1964].

The major recent result has been the measurement of the angular size of the source of the bursts at $19.7 \mathrm{Mc} / \mathrm{s}$. This continues earlier work [Slee and Higgins, 1963] in which the sources were not significantly resolved. The present observations were made near the oppositions of 1963 and 1964, using baselines up to 12,700 wavelengths long. At the longest baselines the sources are well resolved (fig. 1), the apparent diameters ranging from $5 \mathrm{sec}$ of arc to greater than $15 \mathrm{sec}$ of arc. This variability is real.

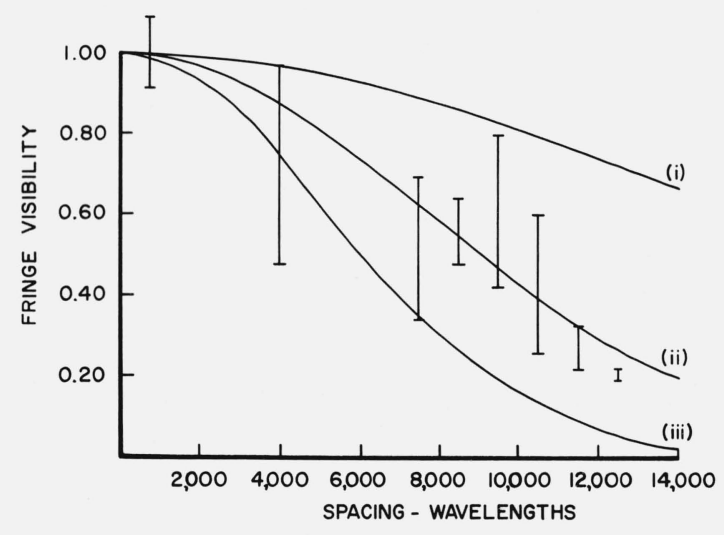

FigurE 1. Ranges of observed Jovian fringe visibility plotted against effective spacing of the interferometer. The curves represent the theoretical fringe visibilities for Gaussian sources of half-brightness sizes (i) $5 \mathrm{sec}$ of arc, (ii) $10 \mathrm{sec}$ of arc, and (iii) $15 \mathrm{sec}$ of arc. 
At periods when the bursts are similar at the two ends of the baseline, the fringes are stable over periods of several minutes. In a typical example, the 42 fringes recorded over a period of several minutes indicated a systematic movement of less than $4 \mathrm{sec}$ of arc, and a maximum scatter of $\pm 3 \mathrm{sec}$ of arc. Thus neither the size of the region in which successive bursts occurred, nor its systematic movement, exceeded 0.1 of the planetary diameter.

Observations of the increase in the angular size of other radio sources seen through the ecliptic suggest that interplanetary scattering can explain the observed angular sizes of the Jupiter bursts, and that the intrinsic size of the bursts is less than the apparent size. It is suggested that the scattering regions are 0.01 to $4.2 \mathrm{~A}$.U. from the Earth. It is furthermore suggested that the apparent burst structure of the Jovian radiation is produced by scintillations occurring within 0.01 A.U. of the Earth.

Note added in proof: The authors now consider that the restriction of the electron irregularities responsible for the scintillations to a region within 0.01 A.U. of the Earth is probably not correct on account of focusing difficulties. In all probability, the observed angular sizes and the burstiness of the radiation are manifestations of a random diffraction process occurring between the Earth and Jupiter, although it is not certain that the same electron irregularities are responsible for both effects.

\title{
References
}

Bigg, E. K. (1964), Influence of the satellite Io on the Jupiter's decametric emission, Nature 203, No. 4949, 1008-1010. Slee, O. B., and Higgins, C. S. (1963), Long baseline interferometry of Jovian decametric radio bursts, Nature 197, 781-782.

\section{Discussion Following Slee and Higgins' Paper}

Frank Drake: J. N. Douglas has recently shown that the direction of drifts observed over several stations reverses at times of Jupiter opposition. This reversal is consistent with the sources of the scintillations moving along with the solar wind outward from the Sun. The inferred scale of structures is hundreds of kilometers, and the speeds are upwards of $1000 \mathrm{~km} / \mathrm{sec}$. This picture is consistent with our picture of interplanetary scintillations observed in small radio sources by Hewish.

\section{Frequency and Polarization Structure of Jupiter's Decametric Emission on a 10-Millisecond Scale}

\author{
James W. Warwick and Mark A. Gordon \\ Astro-Geophysics Department, University of Colorado \\ and
}

High Altitude Observatory, Boulder, Colo.

\begin{abstract}
We present observations of decameter Jupiter emission on a 10-millisecond time base. After describing the swept-frequency polarimeter we discuss polarization and spectral characteristics in terms of propagation conditions along the ray path from Jupiter to Arecibo.
\end{abstract}

Formidable evidence has accumulated during the 10 years of Jupiter decametric observations on the existence and reality of exceedingly short-lived and narrowband phenomena. On time scales from no longer than a few seconds down to a few milliseconds, these structures have been seen with a variety of different techniques, from dynamic spectrographs, through multistation equipment, to wide-baseline interferometers. The major question that has been asked has been the source of the fluctuations: Is it in the terrestrial ionosphere, in interplanetary space, or in or near the source of the radiation at Jupiter itself? The answer given generally has been that the interplanetary medium is responsible, the data thus suggesting fine structure in the solar wind plasma flowing out from the Sun. In fact some of the data (such as time shifts observed between data from multiple stations) support this conclusion, but others do not (for example, failure of correlation at closely spaced stations, and some of the structures on dynamic spectra).

With these problems in mind we decided to investigate the fast-time evolution of the spectrum with as complete a determination of the state of polarization 
of the waves as we could establish. We were fortunate to be able to use the Arecibo 1000 -ft dish for this work, with a broadband, crossed-polarization, log-periodic dipole feed designed by Collins Radio Company for the Air Force Cambridge Research Laboratories. With this combination, Jupiter's decametric emission could be probed over a wide frequency range, with high speed, and satisfactory polarimetry.

A suitable receiver did not exist, and in fact, since we considered that the polarization objectives were very important, we had to invent one for the task.

We had found that terrestrial Faraday effect was a common aspect of our more limited data taken in Boulder with the slow dynamic spectrograph (see fig. 1). The existence of this well-established effect suggested the format for our eventual Arecibo receiver. In the Earth's ionosphere, even at subequatorial latitudes such as that of Puerto Rico, at $30 \mathrm{Mc} / \mathrm{s}$ the propagation modes are quasi-longitudinal over a wide range of $\theta$. Since the terrestrial Faraday effect results from the differential phase path developed between the ordinary and extraordinary modes (which have opposite circular polarization), by observing Jupiter in these modes we can eliminate the effects of our ionosphere. A polarimeter that determines only two intensities cannot establish the complete EM state of the incoming waves. It is necessary by some means to measure the phase difference between the circular modes as well. Furthermore, the intensity of the waves that are uncorrelated between the two circular modes must be established so that we can measure any unpolarized component of the incident radiation.

We have combined these various functions into a single spectrographic receiver in the following way. If we combined the outputs of the two circular antennas, the resultant signal would represent the signal received by a single linear dipole. The position angle of the dipole is determined by the phase difference between the ordinary and extraordinary mode anten-

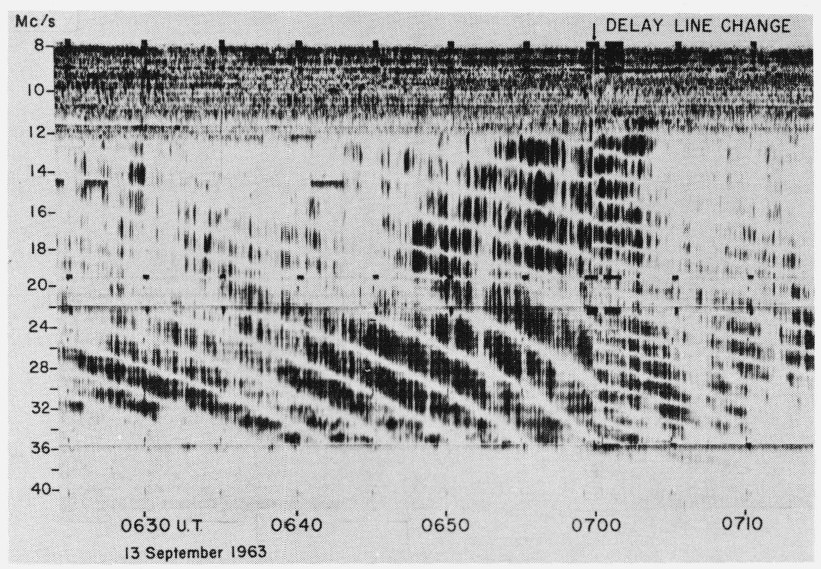

FiguRe 1. Faraday effect in Jupiter's decametric emission (Warwick and Dulk, 1964)

The Faraday fringes are nearly horizontal dark streaks which are widely spaced in frequency at high frequency, $36 \mathrm{Mc} / \mathrm{s}$, but become closely spaced towards $20 \mathrm{Mc} / \mathrm{s}$. The curved diagonal white streaks are interferometer fringes, whose origin is purely instrumental. nas. We actually combine the $\mathrm{O}$ and $E$ modes through a 100-m length of coaxial cable. Therefore, as a function of frequency, the phase difference between $\mathrm{O}$ and $E$ changes linearly in an easily predicted way. The combined $\mathrm{O}$ and $E$ signal therefore represents what would be seen by a spatially rotating dipole making about four complete revolutions as our receiver sweeps from 24 to $36 \mathrm{Mc} / \mathrm{s}$. A compromise is, of course, involved, in that we do not observe the complete state of polarization at all frequencies in this range. We must assume that the polarization is constant over approximately $2 \mathrm{Mc} / \mathrm{s}$ of the swept range (the fringe separation as a function of frequency). As an alternative to assuming constancy of the polarization, we might assume that we know the mechanism that causes the polarization to vary. For example, it might be terrestrial Faraday effect. We might then use this effect to predict what the records should look like. The point is that we do not necessarily neid to know the polarization at intervals as close as $2 \mathrm{Mc} / \mathrm{s}$ or less in order to determine completely the state of the incoming radiation.

Our receiver operates on a time-shared basis between the following configurations: (a) combined (rotating linear dipole); (b) left circular; (c) right circular. The sequence is (a), (b), (a), (c), (a), etc., with each sweep in a given mode requiring $10 \mathrm{msec}$ to complete.

The presentation is on $35-\mathrm{mm}$ film, with the two circular states together occupying about half the width of the film, and the rotating linear display on the other half. There are separate oscilloscopes for each of the two halves, and they are simultaneously photographed on a continuously moving film. With an upper limit of only 0.04 sec for events to be completely resolved in time we are able to record Tau A as a control for sensitivity and standing-wave ratio. In fact, the radio star produces a perfectly smooth broadband record, just as we would expect if our equipment operates properly. This confirms the measurements we have been able to carry out of its performance by laboratory studies of the quadrature hybrid combiner, and field studies (made from the "vertex" of the 1000 -ft dish) of the cross-polarization response of the Collins log-periodic feed. The isolation between the crossed elements exceeds $25 \mathrm{~dB}$.

In virtually all the data we recorded, with but very few exceptions, our equipment did not impose any limitations resulting from the time between sweeps. Out of 19 recorded Jupiter events, taken on a total of 37 nights of observations in October, November, and December 1964, we had only three cases of bursts faster than the 0.04-sec sweep repetition rate. We shall return to the most important of these in a moment.

First of all, however, we will exhibit data typical of the great majority of our observations. Figure 2 shows the linear dipole record at the top, above the two narrower strips from the circular antennas. The middle record is the left-circular state. Note the relative separateness of the individual sweeps from the circular antennas, a result, of course, of the programming of the sweeps. 




$-15$

FIGURE 2. Arecibo swept-frequency polarimeter record at 0154 UT, 10 December 1964.

Each of the three strips covers the range 24 to $37 \mathrm{Mc} / \mathrm{s}$. The total length of the record is about 15 sec (see text tor further description).

This equipment also ran, at much wider bandwidth, on simple dipoles against a ground screen in Boulder. Even on those poorer records the phenomena of this event were clearly visible: slow variations in the intensity, and complex structure in frequency. Especially, note the narrow-bandwidth, high-frequency strip of isolated emission. We should mention in particular that for almost all of these records from Arecibo we have matching records made in Boulder; the identification of these bursts as Jupiter bursts is therefore virtually certain in all cases.

Because we have, in effect, a rotating linear antenna, we can by appropriate choice and location of the delay line in a circular channel, speed up or slow down the Faraday rotation of an eventually fixed polarization ellipse in the record. On the linear strip, one can see the Faraday fringes clearly. Note, for example, their variation in frequency; they are spaced more closely at $24 \mathrm{Mc} / \mathrm{s}$ than at $30 \mathrm{Mc} / \mathrm{s}$. As we had already concluded from the Boulder records of this phenomenon on other occasions, this kind of record is in general completely consistent with the assumption that all of the Faraday effect occurs in the Earth's ionosphere, which is a puzzling conclusion in view of the facts of Jupiter's strong magnetic field and ionosphere.

The two circular states also show strong and variable structure independent of the Faraday effect shown on the linear record. Note, however, that the structure of the circular polarization does appear as modulation on the linear channel. The right-hand signal is consistently stronger than the left-hand, which confirms and extends to high frequency the earlier determinations of the ellipticity and sense of the decametric emission.

Figures 3 and 4 show a detailed comparison of the structure in frequency in the circular states. Despite the complication of the structure, it is in fact identical on the two channels, L and R. The emission therefore is elliptical with roughly constant axial ratio and orientation as a function of frequency above $24 \mathrm{Mc} / \mathrm{s}$. This result is consistent with the previous Boulder result. The striking periodicity in frequency suggests a standing-wave effect, perhaps a combination of direct and reflected waves from Jupiter or perhaps elliptical

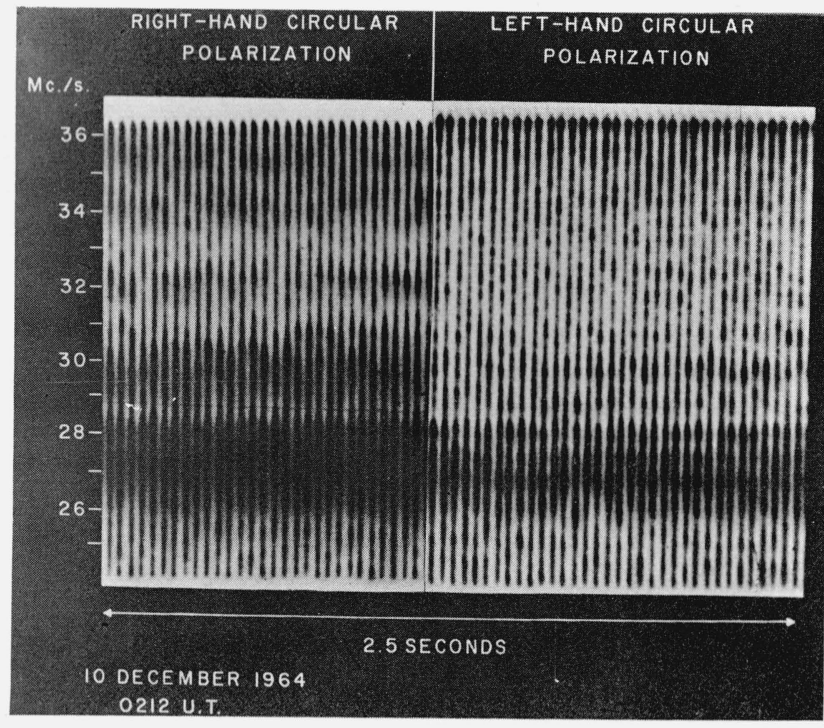

FIGURE 3. A detail of the record from 0212 UT, 10 December 1964 Immediately adjacent records in the $\mathrm{L}$ and $\mathrm{R}$ states are compared (see text).

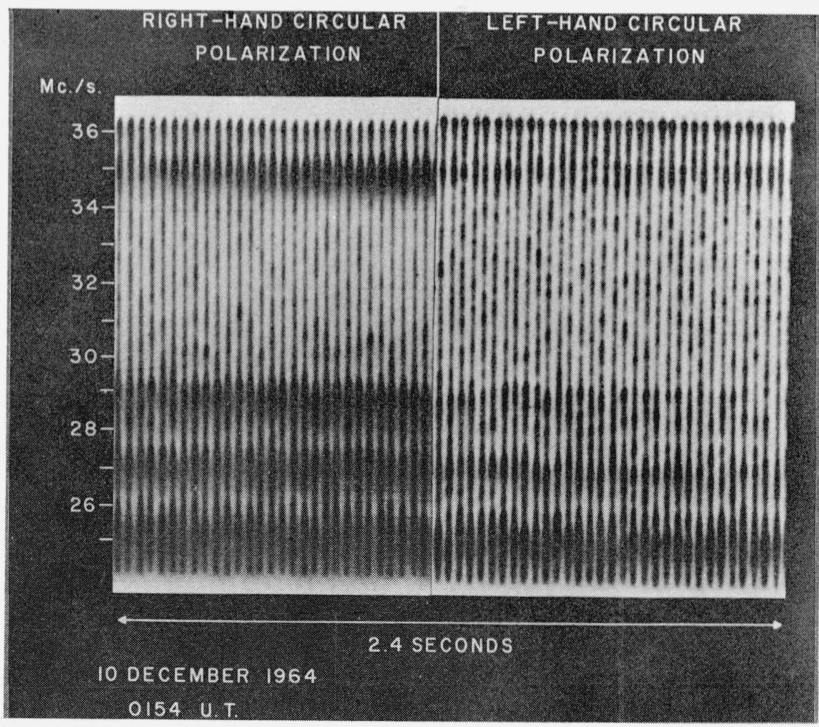

Figure 4. Same as figure 3, but at 0154 UT (see text).

Faraday effect. The effect most likely occurs at Jupiter.

We found an especially persistent case of extremely fast variations on 17 October 1964. Figure 5 shows this event as it appeared in Boulder. Note the sharp high-frequency cutoff above $28 \mathrm{Mc} / \mathrm{s}$, and the narrowband emission at this frequency. Figure 6 shows the early phase of the event at Arecibo, when a steady source appeared together with very rapid variations. Later on, this event had only rapid variations, but we believe that the combination of this early steady source alongside bursts with high-speed modulation, demonstrates that the fast bursts originate in or near Jupiter. 


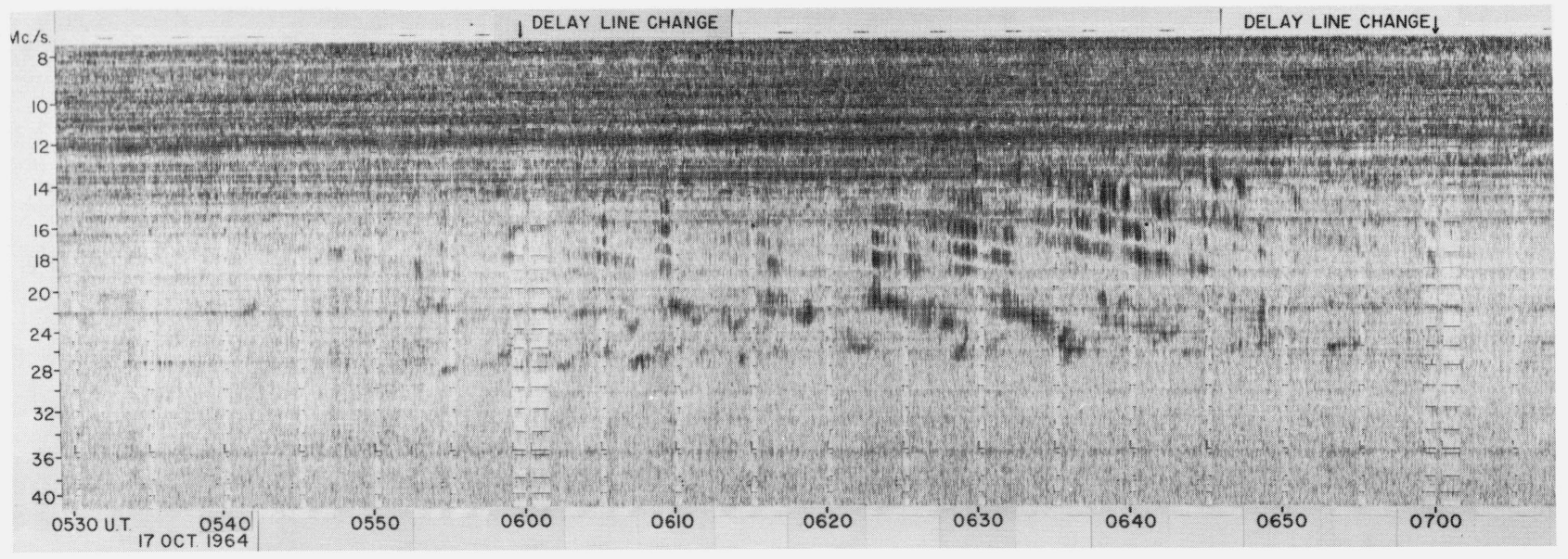

FigURE 5. Boulder spectrogram of Jupiter emission on 17 October 1964 (see text).

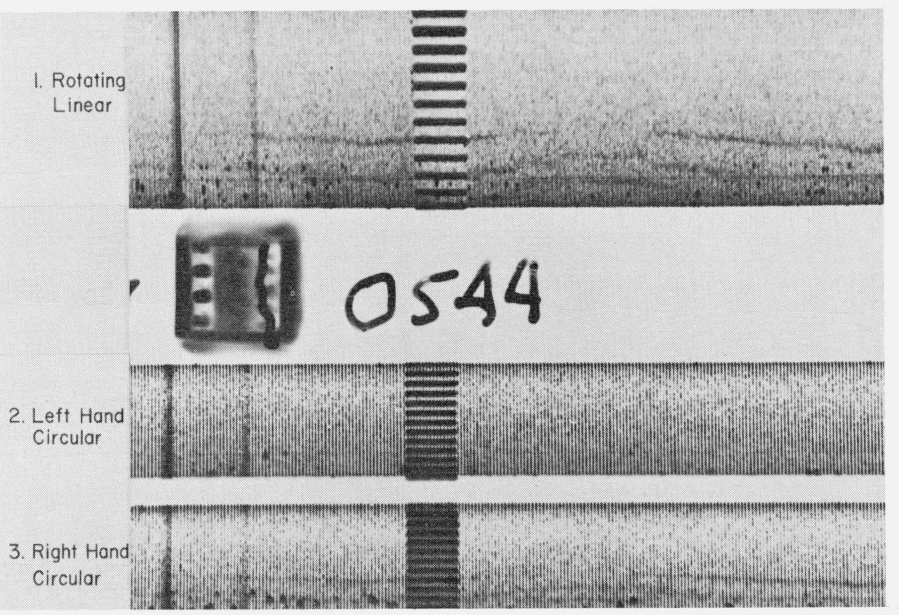

FigURE 6. Arecibo record near 0544 UT, 17 October 1964.

Note the narrowband, but smooth emissions in the form of lanes or paths. These are right-elliptically or circularly polarized, as are indeed the remaining fast bursts that appear on this record.



FigURE 7. 0544 UT, 17 October 1964.

Fast bursts, drifting at $25 \mathrm{Mc} / \mathrm{s}^{2}$, appear alternately on all three traces (see text).
The propagation path from Jupiter to us must be virtually identical for the two kinds of emission. Only at the source itself could such a difference arise.

Figure 7 shows the same event a few minutes later, when there are only high-speed fluctuations, and no steady source.

Figure 8 illustrates the way in which the high-speed burst appears alternately on successive spectral scans in the different polarization modes. Half as many pips appear on the $\mathrm{L}$ and $\mathrm{R}$ channels as a result of the time-sharing scheme. [Note that the burst of figure 7 appear many more times $(8$ or 10 on $L+R)$ than does the idealized drifting burst of our sketch.] A very strong burst that persists during the three sweeps would likely appear, regardless of polarization, on all three channels. Therefore, it is difficult for us to make a detailed statement about the nature of the polarization variations in just one isolated burst. The fre- 
quency-drift rate is another matter; it is in the sense from high to low frequencies, and amounts to 5 to $35 \mathrm{Mc} / \mathrm{s}^{2}$. The bursts obviously occur over and over again with very similar drift rates.

We can, however, speak of the polarization variations in frequency because of the statistical properties of the bursts. Figure 9 shows the 17 October events a few minutes later, when the occurrence of bursts seems to aline the pips, especially on the combined channel. This strongly implies that the polarization of the bursts varies with frequency in a way that is stable in time for, at first, only a few seconds, but then, in later minutes of the event, long periods of time involving literally hundreds or thousands of bursts. We conclude, in other words, that the bursts are strong at a given frequency because the polarization ellipse is parallel to our dipole at that frequency, and not because our receiver happened to be tuned to the particular frequency of the burst at that exact moment. To repeat, the alternatives are that the time of beginning of each burst would have to be correlated with the sweep of the receiver or the burst polarization varies in a consistent way with frequency, but remains almost constant in time.

The $\mathrm{L}$ channel is weaker than the $\mathrm{R}$ channel, in general but not in detail, as can be seen, for example, in figure 10. In fact, when one looks at this record in order to compare the frequencies at which the pips occur, one finds that they are anticorrelated between $\mathrm{L}$ and R (figs. 10 and 11). One cause of such an effect might be the speed with which the bursts flick across our range (mentioned above), which might imply an alternation between $\mathrm{L}$ and $\mathrm{R}$. The difficulty with this explanation is that the bursts line up, clearly on $\mathrm{L}+\mathrm{R}$, and also probably on $\mathrm{L}$ and $\mathrm{R}$ separately.

The peaks of the structure are strongest on the $R$ record; at the time of a burst on the L record, however, the burst is stronger there than the emission simultaneously appearing on the $\mathrm{R}$ record. An average over a few tenths of a second of this kind of record would therefore resemble fixed-frequency polarimetric

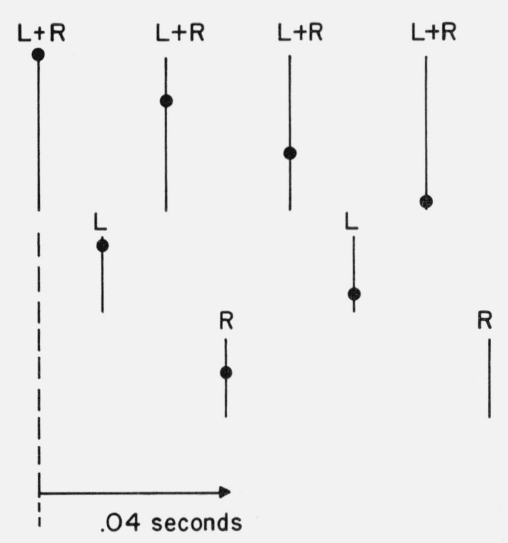

FIGURE 8. Illustration of the way in which the high-speed burst appears alternately on successive spectral scans in the different polarization modes.

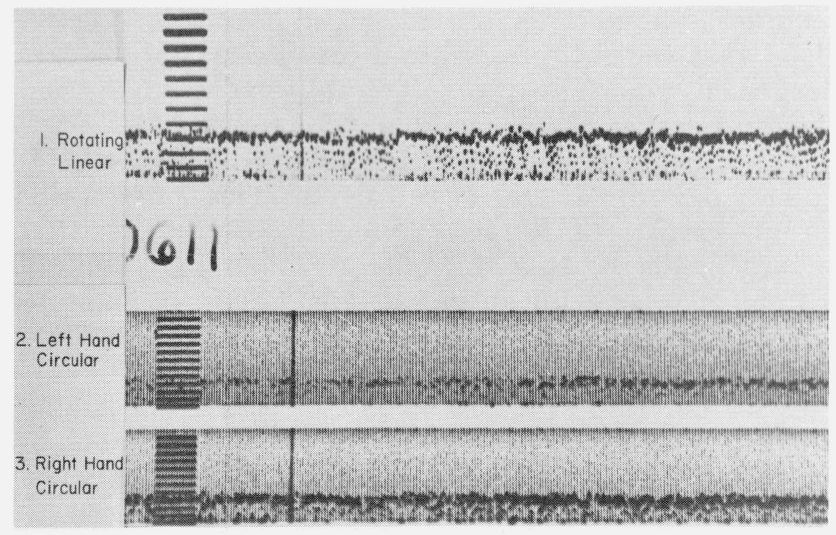

FiguRE 9. 0611 UT, 17 October 1964.

Fast bursts, with quite similar drift rates, occur in alined patterns on the rotating linear trace (see text). 'Warwick, J., and G. Dulk (1964), Faraday rotation on decametric radio emissions from
Jupiter, Science 145, 380-383.
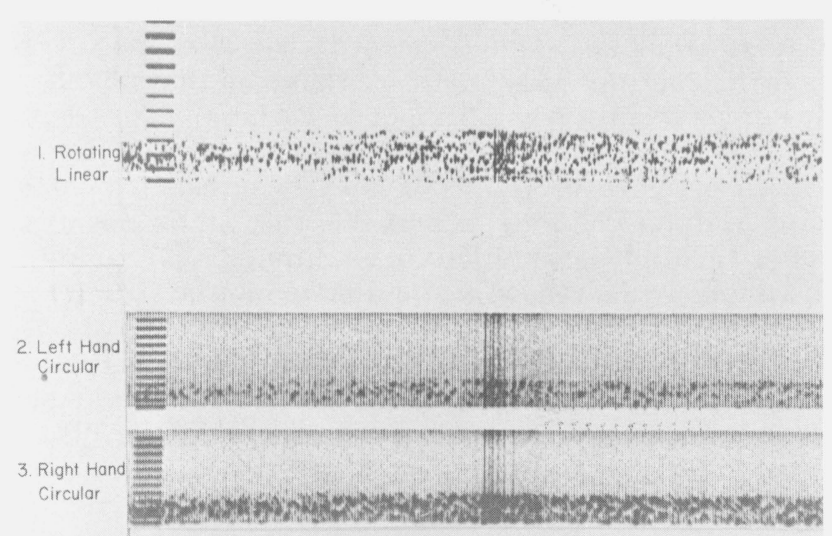

Figure 10. 0615 UT, 17 October 1964.

Fast bursts, showing alinements as in figure 8 ; the burst patterns on the $\mathrm{L}$ and $\mathrm{R}$ traces are anticorrelated (see text, and fig. 10).

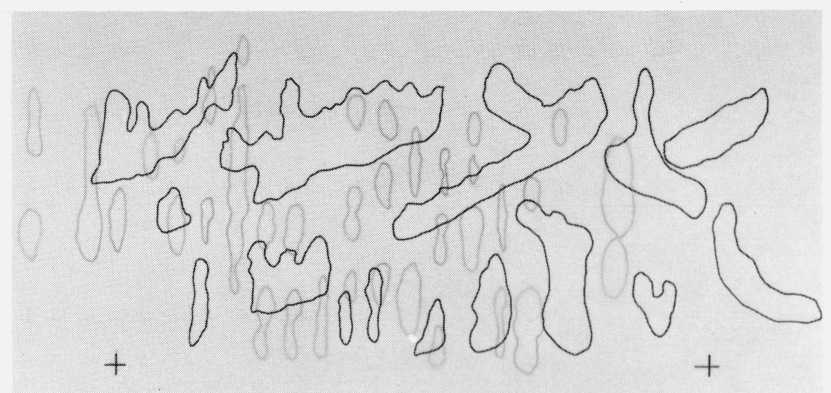

FigURE 11. 0615 UT, 17 October 1964.

A very much enlarged portion of the $L$ and $R$ traces, which have been superimposed. The $\mathrm{R}$ trace is the heavy lines, the $\mathrm{L}$, the lighter ones. 
records as already described in the literature. Note, also, that mixed polarization events as previously described could also result from this kind of fine structure in time and frequency.

It seems probable that the anticorrelation of frequency-periodic $\mathrm{L}$ and $\mathrm{R}$ in this case indicates a different species of Faraday effect from the one that occurs in the Earth's ionosphere. The Jupiter effect distorts a wave with an initial polarization in an elliptical mode into a wave whose polarization sense, axial ratio, and orientation vary periodically with frequency. For this effect to take place the base states describing modal propagation at Jupiter should be orthogonal elliptical modes. Because the $\mathrm{L}-\mathrm{R}$ polarization balance favors $R$, we can conclude that the base modes are elliptical (albeit with large axial ratio) rather than linear. If we measure all parameters of the $L$ and $R$ records we can in principle determine the polarization of the wave that initiates the Faraday effect, and the direction of propagation with respect to the magnetic field.

Figures 6, 7, 9, and 10 show successive phases in the development of Jupiter emission on 17 October 1964. The progression, from clearly separate bursts to swarms of nearly overlapping bursts, suggests that we consider seriously whether Jupiter emission consists of a superposition of myriads of such microbursts. Despite such a record, we also, however, have to confront data such as those at the outset of this day's record, when little or no variation in intensity was to be seen in one component of the emission.

Two or more mechanisms may be present, or perhaps the microbursts represent only a propagation effect, a kind of scintillation in Jupiter's ionosphere. If we follow that reasoning for a moment, the structures might represent wedges or waves in Jupiter's atmosphere. The angular distance through which Jupiter rotates over the duration of a burst would correspond to fine structure in the angular emission pattern from a coherently excited source region on Jupiter. According to this description, the "burst" is in reality emission into a kind of antenna lobe swept past the earth by the rotation of Jupiter.

Jupiter rotates about 0.04 sec of arc per millisecond of time. Suppose a coherently excited source region was big enough (on rare occasions) to extend over a large enough distance that could produce an angular fine structure corresponding to $0.04 \mathrm{sec}$ of arc. There are $2 \times 10^{5}$ sec of arc per radian, so that $0.04 \mathrm{sec}$ of arc implies a distance of $2 \times 10^{5} / 0.04=5 \times 10^{6}$ wavelengths. At $30 \mathrm{Mc} / \mathrm{s}$, this distance is $50,000 \mathrm{~km}$, a substantial fraction of 1 Jupiter radius $(=71,000 \mathrm{~km})$. What we must conclude is that coherent excitation of the emission would have to take place over areas nearly as large as the visible surface of the planet! This may already defeat such a proposal; a shattering defeat would have required a coherence distance larger than the planet. If there were fine structure much shorter than $1 \mathrm{msec}$ it would rule out the mechanism. However, from our data taken at their face value, the region of coherence might be as small as only $5,000 \mathrm{~km}$ (10-msec bursts).

Actually, that planetary-scale phenomena might be involved appears to us as an attractive feature. We note, for example, the tiny size of the satellite Io, yet its longitudinally wide range of influence on the decametric emission. Launched from regions perhaps only a few kilometers in dimension, particles stimulated by Io project through over $400,000 \mathrm{~km}$ of converging lines of force into Jupiter's ionosphere, and these affect a wide range of longitudes.

Discussion Following Warwick and Gordon's Paper

I. Shapiro: What is the strongest evidence for a magenetic field on Jupiter?

Answer: The consistent right-hand polarization of the low-frequency emission, and the substantial angular extent of the decimetric emission.

J. A. Roberts: Slee suggests that the fast variations are imposed in the space between Jupiter and the Earth. How can the mechanism you propose be consistent with his result?

Answer: We have only seen these fast bursts rarely, and there may be more than one mechanism producing this kind of modulation. Our evidence was that simultaneously fast and slow variations appeared in the Jupiter data on 17 October 1964.

(69D12-586) 